\title{
CTRC gene polymorphism (p.G60=; c.180 $C>T)$ in acute pancreatitis
}

\author{
Dorota Koziel ${ }^{1 *}$, Stanislaw Gluszek ${ }^{1,2}$, Artur Kowalik $^{3}$ and Malgorzata Chlopek ${ }^{3}$
}

\begin{abstract}
Background: The aim of the study was to determine the relationship between the presence of p.G60 = polymorphism (c.180C > T; rs497078) CTRC and the incidence together with the clinical course of acute pancreatitis (AP).

Methods: Two hundred ninety-nine people suffering from AP and 417 healthy volunteers were subjected to the study. DNA was isolated from blood samples.

Results: $C T R C$ p.G60 = polymorphism (c.180C $>$ T) occurred more frequently in the AP group $(p=0.015)$. The $C T$ and TT genotype was found in $27.8 \%$ of the AP patients and in $19.9 \%$ of the healthy subjects $(p=0.017)$. No significant correlation was found between having the $C T$ and $T T$ genotype and the severity of the AP clinical course. In 6 patients (2\%) with the CT genotype, a SPINK1 gene mutation was found, while in the control group it was found in 3 patients $(0.7 \%),(p>0.05)$. All patients with the present SPINK1 mutation with the CT genotype had a moderate or a severe course of the disease $(p=0.0007)$.
\end{abstract}

Conclusions: CTRC polymorphism Hetero p.G60=; C.180C > T increases the risk of an AP occurrence and together with the SPINK 1 mutation, may be responsible for a more severe course of the disease.

Keywords: Acute pancreatitis, CTRC polymorphism, Etiology

\section{Background}

In recent years, a higher incidence of acute pancreatitis (AP) has been reported in many countries. Poland is one of the countries with a high incidence rate, which equals $79,7 / 100000[1]$.

It is generally accepted that the direct cause of the disease is the activation of digestive enzymes in the acinar cells. An inflammatory condition in the pancreatic area initiates a cascade of immunological events, which play a significant role in disease development [2]. The course of the disease is usually mild, but in about $20 \%$ of patients, moderate or severe AP develops [1]. The most common causes of AP include cholelithiasis and alcohol consumption, however some studies point to the importance of genetic mutations and polymorphisms [3-6]. Such an approach indicates a complex and multifactorial nature of acute pancreatitis. Genetic factors should be taken into account together with other factors which cause the disease [7]. So far, there is no long term follow-up that

\footnotetext{
* Correspondence: dorota.koziel@wp.pl

${ }^{1}$ Faculty of Medicine and Health Sciences, Jan Kochanowski University, Kielce, Poland

Full list of author information is available at the end of the article
}

would allow us to understand the role of genetic factors during the first onset of AP [8].

Some mutations play an important role in the development of pancreatitis. In normal conditions (genes are not mutated), trypsin in the pancreatic parenchyma is not activated, whereas mutations of genes cause disorders in secretion, enzymes are activated and the mutations predispose the pancreas to pancreatitis. These factors include: the gene coding cationic trypsinogen (PRSSI) and serine peptidase inhibitor Kazal type 1 (SPINK1). Other genes regulating trypsin, such as the independent calcium-sensing receptor gene (CASR), the gammaglutamyltransferase1 gene (GGT1), the cystic fibrosis transmembrane conductance regulator (CFTR) and the recently discovered chymotrypsin $\mathrm{C}$ gene $(C T R C)$, seem to be the disease modifiers with a lower risk. The protein $C T R C$ codes chymotrypsin $\mathrm{C}$, which is produced by the pacreatic acinar cells [7].

Several papers have been published by Sahin-Toth's group elucidating the complex regulatory function of CTRC on trypsinogen activation. Namely, trypsinogen activation in active trypsin is controlled by two processes. 
Firstly, the cleavage of the trypsinogen activation peptide accelerates the autoactivation of trypsinogen. The second mechanism is based on the cleavage of the peptide in the trypsinogen calcium binding loop which promotes its degradation [9-13].

Based on the GTex site (http://www.gtexportal.org/ home/), data concerning the rs497078 SNP QTL, the TT genotype correlated with a lower expression of the CTRC gene in comparison to the CT and CC genotype in normal pancreas. However, it should be mentioned that these results are based on 149 cases with the following genotype distributions CC: 113, CT:33, TT:3. Bearing the above in mind, and taking into consideration the complex nature of, the regulation of trypsinogen activation by $C T R C$, it is possible that the $\mathrm{T}$ allel could be a risk factor for pancreatitis. However, in order to reveal the true function of the $\mathrm{T}$ allel, more studies are needed [14].

The aim of the study was to determine the relationship between the presence of the p.G60=polymorphism (c.180C > T; rs497078, NM_007272.2:c.180C > T), CTRC and the incidence and clinical course of acute pancreatitis in the Polish cohort.

\section{Methods}

The study included 299 patients with acute pancreatitis, being inhabitants of the Kielce Region in Poland who gave their informed consent for the collection of genetic material. The control group consisted of 417 healthy inhabitants who accepted the invitation to take part in the research.

The diagnosis of mild, moderate or severe acute pancreatitis (SAP), according to the Atlanta 2012 classification, was the criterion for inclusion in the study. A diagnosis of AP was based on the joint interpretation of medical history, a physical examination and targeted laboratory tests. The diagnosis was based upon the satisfaction of at least 2 of the following 3 criteria: (1) upper abdominal pain of sudden onset, frequently radiating towards the back; (2) lipase or amylase activity in serum $>3$ times the upper limit of normal; and (3) results of imaging tests that allow one to obtain crosssectional images: computed tomography (CT), nuclear magnetic resonance (NMR), or ultrasonography (USG). In the analysis of the etiology of AP, taking a person's medical history can determine the following: alcohol consumption, the occurrence of concomitant diseases, drug intake, procedures undergone and endoscopic retrograde cholangiopancreatography (ERCP). Biliary etiology was confirmed based on USG, CT, NMR, and/or ERCP, only if there were indications for the performance of these procedures, for example, icterus. Endoscopic ultrasonography was performed in some patients to confirm biliary AP. Alcohol consumption was evaluated with the Short Alcohol Dependence Data (SADD) Questionnaire, and self-estimated alcohol consumption via an interview. Acute pancreatitis was diagnosed if a patient gained $\geq 10$ points on the SADD Questionnaire, or if the period of alcohol abuse lasted $\geq 1$ year, and the daily dose was $40 \mathrm{~g}$ of pure ethanol.

Exclusion criteria were a diagnosis (which took place during interviews) of chronic pancreatitis, as well as the enlargement of the Wirsung duct and calcifications larger than $5 \mathrm{~mm}$, which were diagnosed during transabdominal ultrasonography.

The control group included adult volunteers selected by random sampling, without any apparent concomitant diseases that could have affected the structure and expression of genes to be tested in the study.

The study was approved by the Committee on Bioethics at the Faculty of Medicine and Health Sciences, Jan Kochanowski University in Kielce. Each patient and member of the control group gave their informed consent to genetic testing.

\section{Study samples}

Whole blood samples were submitted to the Department of Molecular Diagnostics (Holycross Cancer Center, Kielce, Poland) for genetic testing.

\section{DNA Extraction}

Whole blood extraction was performed using the Genomic Blood AX Micro Gravity (A \& A Biotechnology, Gdańsk, Poland) purification system, which uses gravity technology. The DNA was extracted manually with a volume of $0.1 \mathrm{ml}$ of blood, then the DNA concentration was measured using the Nano Drop (Thermo Scientific, Waltham, MA, USA).

\section{HRM}

This PCR (forward 5'-CTGACACACAGCCCTCCC -3 'and reverse 5'-ATGGCCAGGTCTCAGGGTAT -3') for the amplification of the target sequence (162 bp) in the CTRC gene were designed using Primer3 web software (RefSeq NM_007272.2).

Similarly, the following primers were (F-TTGCTATGAACTCAAGAATGGAGA;

R-CCGATTTTCAAAACATAACACTG) projected for the amplification of SPINK1.

HRM reactions were performed using reagents from the Qiagen and the Qiagen Rotor Gene machine.

The reactions were carried out using a $15 \mathrm{ul}$ volume according to the following mixture: polymerase $-5 \mathrm{ul}$ (Cat. No. 206546), water -7ul, primer F-1ul (final concentration $0,67 \mathrm{uM}$ ), primer $\mathrm{R}-1 \mathrm{ul}$ (final concentration $0,67 \mathrm{uM})$, DNA - 1ul (20-50 ng) /per sample.

Amplifications were performed using a 5-min initial denaturation at $95{ }^{\circ} \mathrm{C}$ followed by 37 cycles of $10 \mathrm{~s}$ at 
$95{ }^{\circ} \mathrm{C}, 30 \mathrm{~s}$ at $65{ }^{\circ} \mathrm{C}$, with an initial 10 cycles of touchdown $\left(1^{\circ} /\right.$ cycle $)$ and $10 \mathrm{~s}$ at $72{ }^{\circ} \mathrm{C}$.

Then the melting step was carried out in the range of $80^{\circ}$ to $93^{\circ}$ for CTRC, and $75-80{ }^{\circ} \mathrm{C}$ for exon 3 SPINK1. The HRM of studied samples was done in parallel with control WT. Then, all the studied samples were analyzed relative to the control curve (WT).

Genotypes were assigned based on shape differences between curves. The samples with the greatest vertical distance from the WT melting curves were heterozygotes, and melting curves between the heterozygous and WT samples were homozygotes (Fig. 1).

We have comprehensively validated our HRM methodology. 20\% (143 samples) of all the studied samples have been sequenced. The concordance with genotypes assigned based on melting method was $100 \%$. Finally, our results are supported by a similar frequency of mutation detected in a healthy population in comparison to data from the 1000Genome project.

\section{Sequencing}

The curves diverging in shape from the control curve (WT) were verified by applying capillary sequencing. The concentration of DNA in these samples was measured using the electrophoresis device Multina (Shimadzu, Japan). For the Sanger sequencing reaction, PCR amplification products were purified using $10 \mathrm{U}$ (Cat. No. EN 0582) of exonuclease I and $1 \mathrm{U}$ of phosphatase
Fast-AP (Cat. No. EF 0651) (Thermo Fisher). The reaction was incubated for $15 \mathrm{~min}$ at $37{ }^{\circ} \mathrm{C}$, followed by 20 min at $80{ }^{\circ} \mathrm{C}$.

Sequencing reactions were performed using forward and reverse sequence-specific primers (described above) and the ABI-PRISM Big Dye Terminator version 3.1 kit (Applied Biosystems, Foster, CA; Cat. No. 4337450), according to the manufacturer's instruction manual. Sequencing reactions were analyzed using the 3130 Capillary Sequencer (Applied Biosystems, Foster, CA). The generated sequences were compared relative to the reference sequence RefSeq (NM_007272.2), using the NCBI Blast Nucleotide program.

\section{Statistical methods}

Categorical data were expressed as number and percentage distributions. Proportions were compared between groups by the chi-square test or the Fisher's exact test as appropriate. Odds ratios (OR) with 95\% confidence intervals $(95 \% \mathrm{CI})$ were calculated using the univariate logistic regression model. Quantitative data were characterized by medians, and the non-parametric Mann-Whitney $\mathrm{U}$ test was used for comparisons between groups. The two tailed $p$-value $<0.05$ was considered to be statistically significant.

All computations were performed using the statistical package R, version 3.1.2 (R Core Team (2014). R: A language and environment for statistical computing. $\mathrm{R}$ Foundation for Statistical Computing, Vienna, Austria.

Normalised Graph for HRM A.HRM

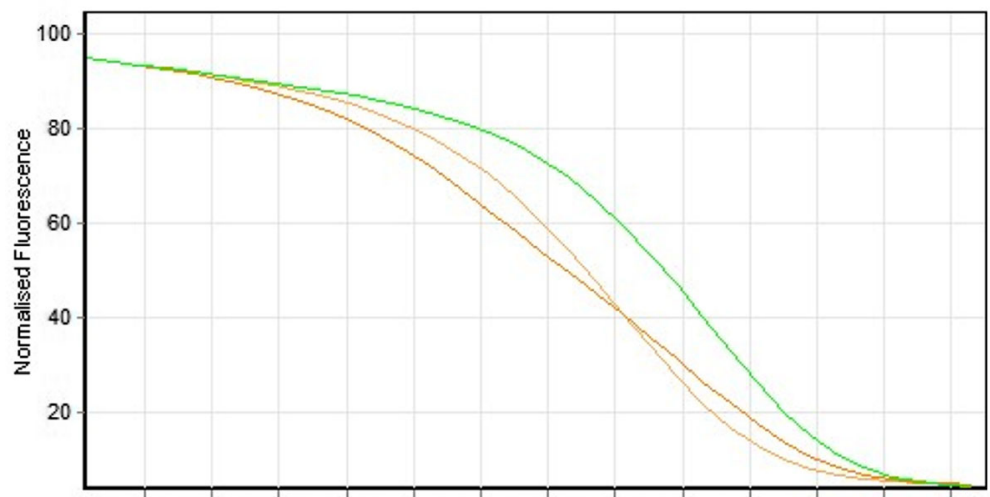

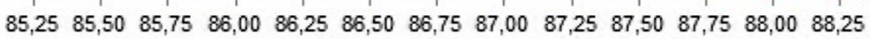
deg.

\begin{tabular}{|l|l|l|l|l|}
\hline \multicolumn{5}{|l|}{ No. Colour Name Genotype Confidence \% } \\
\hline 15 & & Ho & & \\
\hline 16 & & He & & \\
\hline 17 & & WT & & \\
\hline
\end{tabular}

Fig. 1 Example of HRM analysis. Green curve - CC genotype (c.180C), orange - CT genotype (c.180C > T), light orange - TT genotype (c.180 T) 
URL http://www.R-project.org/.) and StatSoft, Inc. (2014). STATISTICA (data analysis software system), version 12. www.statsoft.com.

\section{Results}

The study group included 299 patients who had acute pancreatitis (127 female, 172 male); the median age being 56 years (62 in women, 53 in men). The course of AP was mild in $69.6 \%$ of patients, moderately severe in $13.4 \%$, and severe in $17.0 \%$. The dominant etiological factors of the disease was cholelithiasis (41.8\%) and alcohol $(30.8 \%)$, but in $25.4 \%$ of the patients no other cause than an idiopathic cause of incidence was identified.

The control group consisted of 417 healthy inhabitants of the Kielce Region (271 women and 146 men), with the median age being 46 (46 in women, 48 in men, in a general good state of health).

Both in the test group, as well as in the control group, no differences in the age distribution was observed between the patients with the $\mathrm{CT}$ and $\mathrm{TT}$, versus the CC genotype. In the test group, the median of the patients' age with the $\mathrm{CC}$ genotype was 57 years, and with the CT and TT genotype, 53 years $(p=0.22)$. In the control group, the median age of the patients with the $\mathrm{CC}$ genotype was 47 years, and with the CT and TT genotype, 45 years $(p=0.68)$.

The CT and TT genotype was found in $27.8 \%$ of the AP patients and in $19.9 \%$ of the healthy subjects $(p=0.017)$. The frequency of the TT genotype occurrence was not statistically different between the groups of healthy and sick individuals $(p=0.803)$. However, the CT genotype was significantly more frequent in the AP group compared with the control group $(p=0.015)$ (Table 1).

To reinforce our analysis, we performed a comparison of allelic distribution between our control group and other European populations (Utah residents with Northern and Western European ancestry, Finnish, British, Iberian, Toscani) using data from the 1000 Genomes project [15]. We found that the allelic distribution in our control group is similar to the Toscani and Finish populations
(T:10\%, C:90\%) ( $p=0.518)$, but is different when we take into account the Utah, British and Iberian populations (T:8\%, C:92\%). The genotype distribution was in agreement with the Hardy -Weinberg equilibrium for cases $(p=0.50)$ and controls $(p=0.52)$. The T allele was the minor allele, and was observed more frequently in cases than controls $(14.7 \%$ vs. $10.7 \%, p=0.023)$.

In order to analyze allelic distribution in the studied population, we applied a few genetic models (Table 2.)

With the four models, after applying the Bonferroni corrections to the results of the p-value, less than 0.05 / $4=0.0125$ are found to be statistically significant. It should, however, be kept in mind that this amendment is very conservative, so one can assume that the results of the $p$-value $=0.014$ are on the border of statistical significance. We found that after applying the Bonferroni correction, only the genotypic and dominant model are on the border of statistical significance.

The presence of the CT and TT genotype was not statistically significantly dependent on gender, neither in the test group (women: 38/127 (29.9\%) vs. men: $45 / 172$ [26.2\%], $p=0.56$, test $\mathrm{chi}^{2}$ ), nor in the control group (women: 53/271 [19.6\%] vs. men: 30/146 [20.5\%], $p=0.91$, test $\mathrm{chi}^{2}$ ). The patients' age average with the CT and TT genotype was similar, both in the test group (45.2 vs. 45.5 ) as well as in the control group (54.1 vs. 55.3).

Only in one case of an AP patient with the CT genotype (p.G60=; c.180C > T), was the occurrence of pancreatic disease mentioned in the family history (AP).

No significant dependency was found between having the CT and TT genotype and the severity of the AP clinical course, but in patients with the TT genotype (p.G60=; c.180 T), no severe AP was observed, which is contrary to the patients with the CT genotype (25\% of whom experienced a moderate course of the disease and $27.5 \%$ a severe course of the disease; $p>0.05$ ) (Table 3 ).

The confirmed cause of the incidences was not related to the CT and TT genotypes, except for the cases in which a carcinoma was diagnosed in the AP course. In this group of sick subjects, $50 \%$ of the cases concerned

Table 1 The comparison of the frequency of the occurrence of the CTRC genotype (p.G60=; c.180C, c.180 T, c.180C > T) in the AP patients' group and the control group of healthy individuals

\begin{tabular}{|c|c|c|c|c|c|c|c|c|c|c|}
\hline & \multicolumn{3}{|c|}{ Patients with $\mathrm{AP}$} & \multicolumn{3}{|c|}{ Control group of healthy individuals } & \multirow{3}{*}{$\begin{array}{l}P \\
\text { value }^{*}\end{array}$} & \multirow{3}{*}{$\begin{array}{l}P \\
\text { value** }\end{array}$} & \multirow[t]{3}{*}{ OR } & \multirow[t]{3}{*}{$95 \% \mathrm{C}$} \\
\hline & \multicolumn{3}{|l|}{$N=299$} & \multicolumn{3}{|l|}{$N=417$} & & & & \\
\hline & Female & Male & Totals & Female & Male & Totals & & & & \\
\hline $\begin{array}{l}\text { CTRC Polymorphism } \\
\text { Homo p.G60=; C.180 T }\end{array}$ & $1(0.8 \%)$ & $4(2.3 \%)$ & $5(1.7 \%)$ & $4(1.5 \%)$ & $2(1.4 \%)$ & $6(1.4 \%)$ & 0.042 & 0.803 & 1.2 & $0.4-3.9$ \\
\hline $\begin{array}{l}\text { CTRC Polymorphism } \\
\text { Hetero p.G60=; c.180C }>T\end{array}$ & $37(29.1 \%)$ & $41(23.8 \%)$ & $78(26.1 \%)$ & $49(18.1 \%)$ & $28(19.2 \%)$ & $77(18.5 \%)$ & & 0.015 & 1.6 & $1.1-2.2$ \\
\hline $\begin{array}{l}\text { CTRC Polymorphism } \\
\text { Homo p.G60=; c. } 180 \mathrm{C}\end{array}$ & $89(70.1 \%)$ & $127(73.8 \%)$ & $216(72.2 \%)$ & $218(80.4 \%)$ & $116(79.5 \%)$ & $334(80.1 \%)$ & & 0.014 & 0.6 & $0.5-0.9$ \\
\hline
\end{tabular}

${ }^{*} p$ value for the difference in genotype distribution among AP patients and control group of healthy individuals

**to compare Totals

$A P$ acute pancreatitis, CTRC chymotrypsinogen $C, O R$ odds ratio 
Table 2 Genetic models and allelic distribution in pancreatitis population

\begin{tabular}{lllll}
\hline Genetic model & Comparison & OR & $95 \%$ Cl & $p$-value \\
\hline Allelic & T vs. C & 1.44 & $1.04-2.01$ & 0.023 \\
Genotypic & CT vs. CC & 1.57 & $1.10-2.24$ & 0.014 \\
& $\Pi$ T vs. CC & 1.29 & $0.39-4.27$ & 0.68 \\
Dominant & CT/T vs. CC & 1.55 & $1.09-2.19$ & 0.014 \\
Recessive & $\Pi$ T vs. CT/CC & 1.17 & $0.35-3.85$ & 0.80 \\
\hline
\end{tabular}

patients with the CT genotype (p.G60=; c.180C $>\mathrm{T}$ ). Homo (p.G60=; c.180 T) CTRC polymorphism was found only in $3.3 \%$ of patients with alcohol etiology (Table 3). Alcohol was also a frequent cause of AP relapses in patients with the TT genotype: $2 / 3$ of the cases $(66.7 \%)$ vs. $50.6 \%$ patients with the CC and $38.7 \%$ with the CT genotype.

Recurrent AP (RAP) was more frequent in patients with the $\mathrm{CT}$ and $\mathrm{TT}$ genotype compared with the group without relapses, differently from the CC genotype patients, but statistical significance was not confirmed $(p=0.47)$ (Table 3$)$.

The relationship between the polymorphism of CTRC Hetero p.G60=; c.180 C > T (CT), known as the AP genetic risk factors, the SPINK 1 (p.N34S) gene mutations was analyzed. In six patient (2\%) with the CT genotype, a SPINK1gene mutation was found (p.N34S), while in the control group it was found in 3 patients $(0.7 \%),(p>0.05)$. All patients with the present SPINK1 (p.N34S) mutation with the CT genotype had a moderate or a severe course of the disease $(p=0.0007)$ (Table 4$)$.

A moderate or severe course of the disease was observed in $6 / 6$ of the patients with the SPINK1 (p.N34S) mutation and the CTRC CT genotype vs. 8/15 of the patients with the SPINK1 (p.N34S) mutation and the CC genotype ( $p=0.06$ in the Fisher's exact test). The observed correlation at the edge of statistical significance suggests that increasing the size of the group may confirm a more severe course of the disease in the case of the combination of the SPINK 1 mutation and the CTRC CT genotype.

The SPINK 1 mutation and CTRC polymorphism Hetero p.G60=; c. $180 \mathrm{C}>\mathrm{T}$ were equally frequent among the patients with disease relapses and without ( $2 / 111$ vs. $4 / 188, p=1$ in the Fisher's exact test).

\section{Discussion}

In recent years, molecular studies have allowed us to identify mutations of key genes, including the CTRC gene, which might be crucial in the development of pancreatitis [16].

So far, only a few papers have been published that confirm the relationship of the CTRC gene polymorphism and an increased tendency to chronic pancreatitis, however, there is a lack of research in which patients with acute pancreatitis have taken part. Pathogenic CTRC variants were found both in the patients with $C P$ and in the control group involving healthy individuals, thus, they should be considered risk factors, however, four CTRC variants have been recognized as pathogenic: p.A73T, p.V235I, p.R254W and p.K247_R254del [17].

Many authors observed a significant association of the synonymous variant c.180C > T (p.G60=) with CP [7, 16, $18,19]$. In the Indian studies, the variant c. $180 \mathrm{C}>\mathrm{T}$ (p.G60=) was found in 23-29\% of the subject population. It increased the incidence risk by about 2.5 times in heterozygotes and tenfold in homozygotes [19]. In our paper, the analysis of the frequency distribution of the CTRC gene genotypes revealed a statistically significant correlation between the frequency of the CT (c.180C > T,

Table 3 The frequency of the occurrence of the CTRC gene polymorphisms (p.G60=; c.180C > T) in the acute pancreatitis patients depending on the severity of the clinical course, etiology and disease relapses

\begin{tabular}{|c|c|c|c|c|c|c|c|}
\hline $\begin{array}{l}\text { Analysis p.G60 = } \\
(\text { c. } 180 \mathrm{C}>\mathrm{T} ; \text { rs } 497078)\end{array}$ & $\begin{array}{l}\text { No. } \\
299\end{array}$ & $\prod_{5}^{\pi}$ No. & CT No. 78 & CC No. 216 & $\begin{array}{l}\text { TT vs. CC } \\
P \text { value }\end{array}$ & $\begin{array}{l}C T \text { vs. } C C \\
P \text { value }\end{array}$ & $\begin{array}{l}\text { TT/CT vs. CC } \\
P \text { value }\end{array}$ \\
\hline \multicolumn{8}{|c|}{ Severity of the course } \\
\hline Mild AP & 208 & $5(2.4 \%)$ & $54(26 \%)$ & 149 (71.6\%) & 0.801 & 0.979 & 0.908 \\
\hline Moderate AP & 40 & - & $10(25 \%)$ & $30(75 \%)$ & & & \\
\hline Severe AP & 51 & - & $14(27.5 \%)$ & 37 (72.5\%) & & & \\
\hline \multicolumn{8}{|l|}{ Etiology of AP } \\
\hline Alcohol & 92 & $3(3.3 \%)$ & $21(22.8 \%)$ & $68(73.9 \%$ & 0.418 & 0.521 & 0.661 \\
\hline Gallstones & 125 & $2(1.6 \%)$ & $33(26.4 \%)$ & $90(72 \%)$ & & & \\
\hline Idiopathic & 76 & & $21(27.6 \%)$ & 55 (72.4\%) & & & \\
\hline Cancer & 6 & & $3(50 \%)$ & $3(50 \%)$ & & & \\
\hline \multicolumn{8}{|l|}{ Reccurrences of AP } \\
\hline Yes & 111 & $3(2.7 \%)$ & 31 (27.9\%) & 77 (69.4\%) & 0.355 & 0.584 & 0.473 \\
\hline
\end{tabular}


Table 4 The description of the AP patients with the confirmed SPINK 1 (p.N34S) mutation and CTRC polymorphism hetero p.G60=; c.180C > T (CT)

\begin{tabular}{|c|c|c|c|c|c|c|c|c|c|c|}
\hline \multicolumn{3}{|c|}{ Severity of the course } & \multicolumn{2}{|c|}{ Reccurrences of AP } & \multicolumn{4}{|c|}{ Etiology of AP } & \multicolumn{2}{|c|}{ Pancreatic diseases in family } \\
\hline Mild AP & Moderate AP & Severe AP & Yes & No & Alcohol & Gallstones & Idiopathic & Cancer & Yes & No \\
\hline Patient No & \multicolumn{10}{|c|}{ SPINK 1 mutation and CTRC Polymorphism Hetero p.G60=; C.180C > T (CT) $n=6 / 299(2 \%)$} \\
\hline 1 & $x$ & & & $x$ & & $x$ & & & & $x$ \\
\hline 2 & $x$ & & & $x$ & & $x$ & & & & $x$ \\
\hline 3 & $x$ & & $x$ & & $x$ & & & & & $x$ \\
\hline 4 & - & $x$ & & $x$ & & & $x$ & & & $x$ \\
\hline 5 & $x$ & & $x$ & & & & & $x$ & & $x$ \\
\hline 6 & & $x$ & & $x$ & & $x$ & & & $x$ & \\
\hline
\end{tabular}

AP acute pancreatitis, CTRC chymotrypsinogen C, SPINK 1 pancreatic secretory trypsin inhibitor gene

p.G0=) genotype occurrence and developing acute pancreatitis $(p<0.05)$ with the adjusted odds ratio $1.6(95 \% \mathrm{CI}$ 1.1-2.2). What's more, applying the four genetic models (Table 2), we have found that none of them have statistical significance. However, genotyping (TT vs. CC) and the dominant model (CT/TT vs. CC) are on the border of statistical significance.

Acute pancreatitis, especially recurring and chronic pancreatitis, should be treated as a complex disorder, in which various genetic and environmental factors can be of significance, which, when they co-occur, are responsible for increasing the risk of the disease development, a change in its character or a growing intensity of the developing process [20]. Tremblay et al. [21] conducted research in a group of 38 patients with acute pancreatitis and severe abdominal pain, as well as hypertriglyceridemia (Lipoprotein Lipase Deficiency- LPLD) and 100 healthy individuals (control). As a result of sequencing the CTRC and SPINK1 genes, they found a positive correlation between the frequency of rehospitalization and a combination of the gene variants: rs545634 (IVS) (CTRC) rs11319 (UTR-3') (SPINK1) [OR=41.4 (CI:2.0-848.0); $p=0.016$ ]. LaRusch et al. [5] analyzed the influence of the variants CTRC p.G60 (c.180 T) on the risk of developing $\mathrm{CP}$ and RAP in a group of patients of European origin. Alcohol and tobacco smoking usually co-occurred, but the frequency of CTRC c.180 T in CP, but not in RAP, was higher among non-drinking smokers (10,8\%), which suggests that smoking, not alcohol, may play a more significant role in this dependency. A frequent variant CTRC c.180 $\mathrm{T}$ may have an influence on the modifications of progression from RAP to $C P$, especially in patients with the CFTR or SPINK1 mutations, who drink alcohol or smoke tobacco [5].

The studies on NAPS2 $=$ CV showed that in the group of 521 patients with $\mathrm{CP}$, there were $25,7 \%$ of patients who had never had AP. A higher percentage of patients with RAP never consumed alcohol or smoked cigarettes compared to the CP and control groups [5]. In our study, alcohol was the dominant cause of developing the disease in patients with polymorphism Homo c.180 T CTRC (60\%). Alcohol was also a frequent cause of the AP relapses in patients with the c.180 T genotype (66,7\%).

LaRusch et al. [5] found that CTRC c.180C > T alleles significantly increase the $\mathrm{CP}$ risk, but not RAP, which corresponds to the results of our study, in which no correlation was found between the disease relapses with CTRC c. $180 \mathrm{C}>\mathrm{T}$ and polymorphism Homo (c.180 T; p.G60=). In our study we did not confirm the relationship between CTRC c.180C > T and the SPINK 1, CTRC polymorphisms and the frequency of AP development, recurrence and an identified cause of the disease. LaRusch et al. [5] and Masson et al. [22] reported such a dependency in reference to the patients with CP. However, a new finding was the fact that the mutation SPINK1 (hetero p.N34S), in combination with CTRC c. $180 \mathrm{C}>\mathrm{T}$, can be responsible for a more severe course of acute pancreatitis. Due to the small number of patients (6 people), one cannot formulate a decisive conclusion and thus further research is necessary in order to confirm this dependency.

There is a growing number of studies which reveal that genetic factors together with environmental factors may increase the risk of developing pancreatitis.

\section{Conclusions}

CTRC polymorphism Hetero p.G60=; c.180C $>$ T increases the risk of developing acute pancreatitis, and in combination with the SPINK1 mutation, can be responsible for a more severe course of the disease.

\section{Abbreviations}

AP: Acute pancreatitis; ASA-PCR: Allele-specific PCR; BMI: Body mass index; CASR: The calcium-sensing receptor gene; CFTR: The cystic fibrosis transmembrane conductance regulator gene; CLDN2: The protein claudin-2; CP: Chronic pancreatitis; CPA: Carboxypeptydases A variants; CT: Images: computed tomography; CTRC: The chymotrypsinogen gene; ERCP: Endoscopic retrograde cholangiopancreatography; GGT1: Gammaglutamyltransferase1 gene; HRM: High resolution melting; ICP: Idiopathic chronic pancreatitis; NMR: Nuclear magnetic resonance; PRSS1: Cationic trypsinogen gene; PRSS2: Anionic trypsinogen; RAP: Recurrent acute pancreatitis; SADD: Short Alcohol Dependence Data; SAP: Severe acute pancreatitis; SPINK1: The pancreatic secretory trypsin inhibitor gene; USG: Ultrasonography. 


\section{Acknowledgements}

Not applicable.

\section{Funding}

The study was financed from the resources for statutory research at the Jan Kochanowski University in Kielce (615 516).

\section{Availability of data and materials}

The data supporting our findings can be found at: https://zenodo.org/ record/48778.

\section{Authors' contributions}

DK and SG were responsible for the project's concept, patients' recruitment, and collection of clinical data. DK, SG and AK designed the experiment, interpreted genetic and clinical data, and wrote the manuscript. AK designed the genetic analysis, assisted by MC. MC conducted the genetic studies, which were analyzed and interpreted by AK and MC. All authors read and approved the final manuscript.

\section{Competing interests}

The authors declare that they have no competing interests.

We guarantee that this paper is original and has not been published in any form to-date, nor is it under consideration by any other editorial boards.

\section{Consent for publication}

Informed consent was taken from the patients prior to enrolment. As manuscript does not contain any individual person's data in any form (including individual details, images or videos), so consent for publication was not required.

\section{Ethics approval and consent to participate}

The study was approved by the Committee on Bioethics at the Faculty of Medicine and Health Sciences, Jan Kochanowski University in Kielce (Decision No. 27/2010)

All patients recruited gave written consent for the study.

\section{Author details}

${ }^{1}$ Faculty of Medicine and Health Sciences, Jan Kochanowski University, Kielce Poland. ${ }^{2}$ Clinic General Oncological and Endocrinological Surgery, Regional Hospital, Kielce, Poland. ${ }^{3}$ Department of Molecular Diagnostics, Holy Cross Cancer Centre, Kielce, Poland.

Received: 6 April 2016 Accepted: 22 December 2016

Published online: 17 January 2017

\section{References}

1. Gluszek S, Koziel D. Prevalence and progression of acute pancreatitis in the Swietokrzyskie Voivodeship population. Pol Przegl Chir. 2012:84(12):618-25.

2. Bhatia M, Wong FL, Cao Y, Lau HY, Huang J, Puneet P, Chevali L. Pathophysiology of acute pancreatitis. Pancreatology. 2005;5(2-3):132-44.

3. Koziel D, Gluszek S, Kowalik A, Chlopek M, Pieciak L. Genetic mutations in SPINK1, CFTR, CTRC genes in acute pancreatitis. BMC Gastroenterol. 2015;15:70.

4. Solomon S, Whitcomb DC. Genetics of pancreatitis: an update for clinicians and genetic counselors. Curr Gastroenterol Rep. 2012;14(2):112-7.

5. LaRusch J, Lozano-Leon A, Stello K, Moore A, Muddana V, O'Connell M, Diergaarde B, Yadav D, Whitcomb DC. The Common Chymotrypsinogen C (CTRC) Variant G60G (C.180 T) Increases Risk of Chronic Pancreatitis But Not Recurrent Acute Pancreatitis in a North American Population. Clin Transl Gastroenterol. 2015:6:e68.

6. Lee JK, Enns R. Review of idiopathic pancreatitis. World J Gastroenterol. 2007;13(47):6296-313.

7. Ravi Kanth V, Nageshwar Reddy D. Genetics of acute and chronic pancreatitis: An update. World J Gastrointest Pathophysiol. 2014;5(4):427-37.

8. Khurana V, Ganguly I. Recurrent acute pancreatitis. JOP. 2014;15(5):413-26.

9. Szabo A, Sahin-Toth M. Determinants of chymotrypsin C cleavage specificity in the calcium-binding loop of human cationic trypsinogen. FEBS J. 2012: 279(23):4283-92

10. Nemoda Z, Sahin-Toth M. Chymotrypsin C (caldecrin) stimulates autoactivation of human cationic trypsinogen. J Biol Chem. 2006;281(17):11879-86.
11. Szmola R, Sahin-Toth M. Chymotrypsin C (caldecrin) promotes degradation of human cationic trypsin: identity with Rinderknecht's enzyme Y. Proc Nat Acad Sci U S A. 2007;104(27):11227-32.

12. Szabo A, Sahin-Toth M. Increased activation of hereditary pancreatitisassociated human cationic trypsinogen mutants in presence of chymotrypsin C. J Biol Chem. 2012;287(24):20701-10.

13. Geisz A, Hegyi P, Sahin-Toth M. Robust autoactivation, chymotrypsin C independence and diminished secretion define a subset of hereditary pancreatitis-associated cationic trypsinogen mutants. FEBS J. 2013; 280(12):2888-99.

14. Consortium GT. The Genotype-Tissue Expression (GTEx) project. Nat Genet. 2013;45(6):580-5.

15. Gheorghe CP, Mohan S, Oberg KC, Longo LD. Gene expression patterns in the hypoxic murine placenta: a role in epigenesis? Reprod Sci. 2007; 14(3):223-33.

16. Rosendahl J, Witt H, Szmola R, Bhatia E, Ozsvari B, Landt O, Schulz HU, Gress TM, Pfutzer R, Lohr M, et al. Chymotrypsin C (CTRC) variants that diminish activity or secretion are associated with chronic pancreatitis. Nat Genet. 2008;40(1):78-82.

17. Beer S, Zhou J, Szabo A, Keiles S, Chandak GR, Witt H, Sahin-Toth M. Comprehensive functional analysis of chymotrypsin C (CTRC) variants reveals distinct loss-of-function mechanisms associated with pancreatitis risk. Gut. 2013;62(11):1616-24

18. Matas-Cobos AM, Redondo-Cerezo E, Alegria-Motte C, Martinez-Chamorro A Saenz-Lopez P, Jimenez P, Jimenez MR, Gonzalez-Calvin JL, de Teresa J, Osuna FR. The role of Toll-like receptor polymorphisms in acute pancreatitis occurrence and severity. Pancreas. 2015;44(3):429-33.

19. Paliwal S, Bhaskar S, Mani KR, Reddy DN, Rao GV, Singh SP, Thomas V, Chandak GR. Comprehensive screening of chymotrypsin C (CTRC) gene in tropical calcific pancreatitis identifies novel variants. Gut. 2013;62(11):1602-6.

20. Whitcomb DC. Genetics of alcoholic and nonalcoholic pancreatitis. Curr Opin Gastroenterol. 2012;28(5):501-6.

21. Tremblay K, Dubois-Bouchard C, Brisson D, Gaudet D. Association of CTRC and SPINK1 gene variants with recurrent hospitalizations for pancreatitis or acute abdominal pain in lipoprotein lipase deficiency. Front Genet. 2014;5:90.

22. Masson E, Chen JM, Scotet V, Le Marechal C, Ferec C. Association of rare chymotrypsinogen C (CTRC) gene variations in patients with idiopathic chronic pancreatitis. Hum Genet. 2008;123(1):83-91.

\section{Submit your next manuscript to BioMed Central and we will help you at every step:}

- We accept pre-submission inquiries

- Our selector tool helps you to find the most relevant journal

- We provide round the clock customer support

- Convenient online submission

- Thorough peer review

- Inclusion in PubMed and all major indexing services

- Maximum visibility for your research

Submit your manuscript at www.biomedcentral.com/submit
Biomed Central 